УДК 517.9, 519.6

MSC 76M50, 80M40

\title{
SOLVABILITY OF HOMOGENIZED PROBLEMS WITH CONVOLUTIONS FOR WEAKLY POROUS MEDIA
}

\author{
G. V. Sandrakov, A. L. Hulianytskyi
}

Faculty of Computer Science and Cybernetics, Taras Shevchenko Kiev National University, Kiev, Ukraine, E-mail: \{gsandrako, andriyhul\}@gmail.com

\section{РАЗРЕШИМОСТЬ ОСРЕДНЕННЫХ ЗАДАЧ В СВЕРТКАХ ДЛЯ СЛАБО ПОРИСТЫХ СРЕД}

\section{Г. В. САНДРАКОВ, А. Л. ГУЛЯНИЦКИЙ}

Факультет компьютерных наук и кибернетики, Киевский национальный университет имени Тараса Шевченко, Киев, Украина, E-mail: \{gsandrako, andriyhul\}@gmail.com

ABSTRACT. Initial boundary value problems for nonstationary equations of diffusion and filtration in weakly porous media are considered. Assertions about the solvability of such problems and the corresponding homogenized problems with convolutions are given. These statements are proved for general initial data and inhomogeneous initial conditions and are generalizations of classical results on the solvability of initial-boundary value problems for the heat equation. The proofs use the methods of a priori estimates and the well-known Agranovich-Vishik method, developed to study parabolic problems of general type.

KEYWORDS: initial-boundary problems, problems with convolutions, homogenization, homogenized problems, solvability, a priori estimates, Laplace transform.

АннотАция. Рассматриваются начально-краевые задачи для нестационарных уравнений диффузии и фильтрации в слабо пористых средах. Приведены утверждения о разрешимости таких задач и соответствующих осредненных задач в свертках. Эти утверждения, обобщающие классические результаты о разрешимости начально-краевых задач для уравнения теплопроводности, доказаны для общих исходных данных и неоднородных начальных условиях. В доказательствах используются методы априорных оценок и известный метод Аграновича-Вишика, разработанный для изучения параболических задач общего типа.

КЛЮчЕВыЕ словА: начально-краевые задачи, задачи в свертках, осреднение, осредненные задачи, разрешимость, априорные оценки, преобразование Лапласа. 


\section{ВВЕДЕНИЕ}

Математическое моделирование динамических процессов диффузии и фильтрации жидкостей в пористых средах является актуальным при планировании использования подземных ресурсов, разработке методов предотвращения техногенных загрязнений подземных вод и поиска способов очистки таких вод от загрязнений. Исследования таких процессов инженерными методами наблюдений являются дорогостоящими и практически не возможны, ввиду необходимости устанавливать большое количество датчиков на больших территориях и различных глубинах для исследования динамики перемещения жидкостей в реальной пористой среде. Таким образом, моделирование является единственной возможностью прогнозирования и возможной оптимизации методов рациональной вододобычи, очистки и предотвращения загрязнений подземных вод.

Для моделирования процессов диффузии и фильтрации в пористых средах естественно прежде выбрать некоторую модель такой среды. Наиболее просто моделируются пористые среды, имеющие периодическое строение, поскольку для описания таких сред достаточно определить только структуру ячейки периодичности и далее продолжить такую ячейку периодическим образом с подходящими периодами.

Здесь будут рассмотрены пористые периодические среды, образованные большим количеством «блоков», имеющих слабую проницаемость, и разделенных связной системой «разломов» с высокой проницаемостью. Такие пористые среды естественно назвать слабо пористыми. Учет строения таких сред при моделировании приводит к зависимости рассматриваемых уравнений и их коэффициентов от малого параметра, характеризующего микромасштабность пористой среды и проницаемость блоков.

Такие модели для уравнений с одним и несколькими малыми параметрами и периодическими коэффициентами исследовались в [1-5]. Дальнейшие обобщения методов исследования этих моделей для задач диффузии и фильтрации жидкостей, имеющих несколько фаз, приведены в [6-11]. В этих работах получены осредненные начально-краевые задачи в свертках, решения которых приближают решения исходной начально-краевой задачи для слабо пористых сред, и доказаны оценки точности таких приближений и утверждения о разрешимости осредненных задач.

Эти результаты получены в предположении гладкости исходных данных и однородных начальных условиях. В данной работе будет доказана разрешимость таких осредненных задач в свертках при общих исходных данных и неоднородных начальных условиях. Свойства решений таких задач близки к свойствам решений задач переменных порядков, дальнейшие подробности о которых и подходящую библиографию можно найти в [12]. В механике и физике такие задачи в свертках принято называть динамическими задачами с памятью [13]. Значительно более общие осредненные задачи были получены в [14-16]. Однако, такие осредненные задачи являются двухмасштабными и содержат как быстрые так и медленные переменные. В рассматриваемые здесь задачах такие переменные разделены и осредненные уравнения зависят только от медленных переменных. 
Для исследования разрешимости осредненных задач с памятью в данной работе, как и в [1-5], будет использован метод преобразования Лапласа, разработанный в [17] для изучения параболических задач общего типа. Используемые здесь определения из [17] приведены в параграфе 2. Исходная задача о диффузии и фильтрации для слабо пористых сред сформулирована в следующем параграфе. Утверждения о разрешимости осредненных задач в свертках приведены и доказаны в параграфе 4, где использованы априорные оценки решений, полученные в параграфе 3.

\section{1. НАЧАЛЬНО-КРАЕВЫЕ ЗАДАЧИ ДЛЯ СЛАБО ПОРИСТЫХ СРЕД}

Пусть для $n \geq 2$ заданы ограниченная область $\Omega \subset \mathbb{R}^{n}$ и функции $f \in L^{2}\left(0, \infty ; H^{-1}(\Omega)\right)$ и $w \in L^{2}(\Omega)$. Здесь и далее используются пространства функций, определения которых приведены, например, в [13]. Зададим функцию $u=u(t, x)$ как решение начально-краевой задачи

$$
\begin{aligned}
& u_{t}^{\prime}-\operatorname{div} A^{\varepsilon}(\nabla u)=f \quad \text { в } \quad \Omega \times(0, \infty), \\
& \left.u\right|_{t=0}=w \quad \text { в } \Omega, \quad u=0 \quad \text { на } \partial \Omega \times(0, \infty),
\end{aligned}
$$

зависящей от малого параметра $\varepsilon$ следующим образом.

Пусть $F_{1}$ является открытым связным 1-периодическим (периодическим с периодом 1 по каждой из независимых переменных $\left.x_{1}, \ldots, x_{n}\right)$ подмножеством из $\mathbb{R}^{n}$ с локально липшицевой границей, $F_{0}=\mathbb{R}^{n} \backslash \bar{F}_{1}$ является множеством с локально липшицевой границей и

$$
F_{1}^{\varepsilon}=\varepsilon F_{1}=\left\{\varepsilon x: x \in F_{1}\right\}, \quad F_{0}^{\varepsilon}=\varepsilon F_{0}=\left\{\varepsilon x: x \in F_{0}\right\} .
$$

Таким образом, множества $F_{1}$ и $F_{0}$ с общей границей $\partial F_{1}$ вполне определяются множествами $Y_{1}=F_{1} \cap Y$ и $Y_{0}=F_{0} \cap Y$ с границей $\Gamma_{1}=\partial F_{1} \cap Y$, где $Y=(0,1)^{n}$ обозначает ячейку периодичности. Так определенные множества $Y_{1}$ и $Y_{0}$ разбивают ячейку периодичности $Y$ на два множества, соответствующих блокам и разломам, разделенными общей границей $\Gamma_{1}$. Примеры, иллюстрирующие такие разбиения, приведены на рисунке 1.
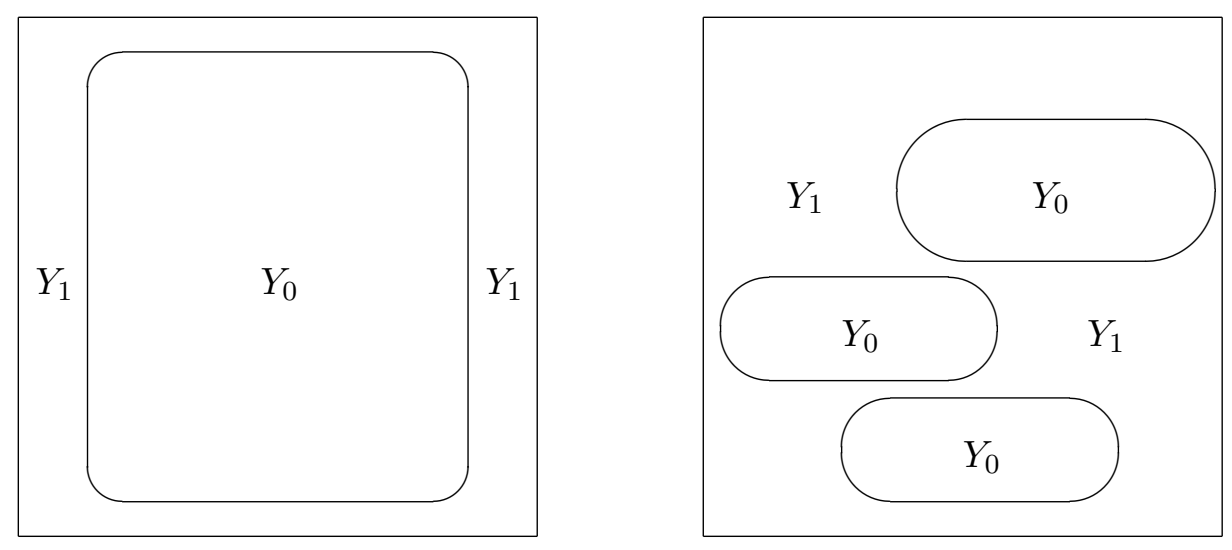

Рисунок 1. Модели разбиения ячейки на блоки и разломы 
Множества $F_{0}^{\varepsilon}$ и $F_{1}^{\varepsilon}$ для фиксированного $\varepsilon$ определяют естественным образом пористые среды с периодической структурой $\Omega_{0}^{\varepsilon}=F_{0}^{\varepsilon} \cap \Omega$ и $\Omega_{1}^{\varepsilon}=$ $F_{1}^{\varepsilon} \cap \Omega$, ограниченные границей $\partial \Omega$ множества $\Omega$, на котором и рассматривается задача (1). Примеры таких сред приведены на рисунке 2.
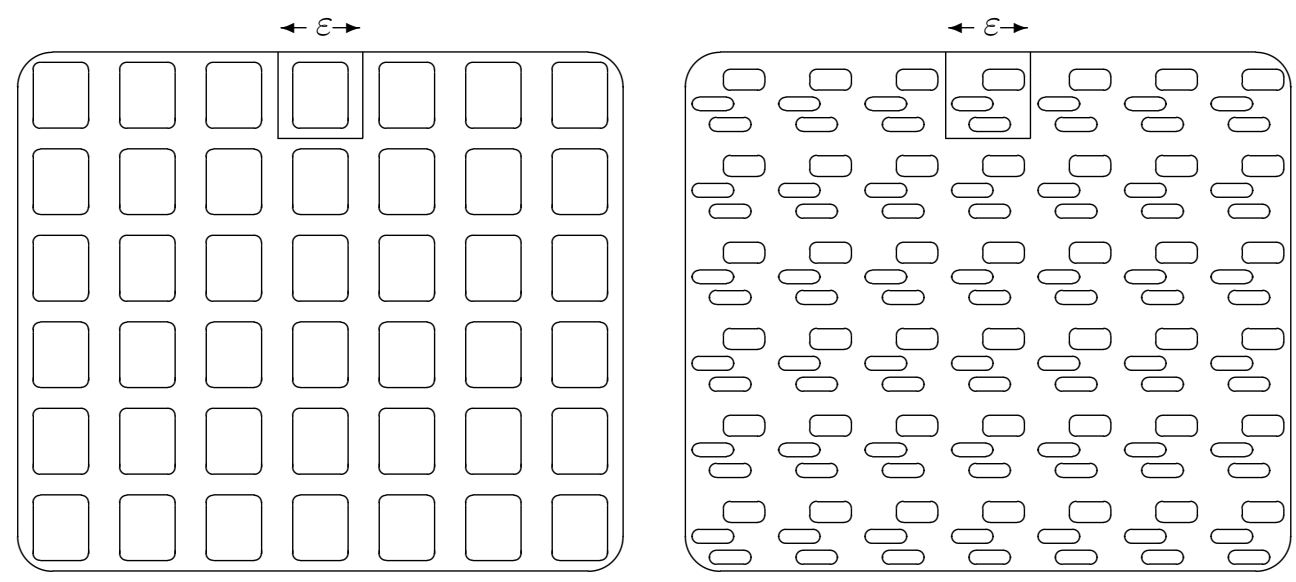

Рисунок 2. Модели пористых сред

Для так определенных моделей пористых сред $\Omega_{0}^{\varepsilon}$ и $\Omega_{1}^{\varepsilon}$, соответствующих блокам и разломам в области $\Omega$, зависимость коэффициентов задачи (1) от параметра $\varepsilon$ задается следующими равенствами

$$
A^{\varepsilon}=\varepsilon^{2} A_{0} \quad \text { в } \quad \Omega_{0}^{\varepsilon} \quad \text { и } \quad A^{\varepsilon}=A_{1} \quad \text { в } \quad \Omega_{1}^{\varepsilon},
$$

где матрицы $A_{0}$ и $A_{1}$ с постоянными компонентами являются симметричными и эллиптическими. Предполагается, что множества $Y_{1}$ и $Y_{0}$ имеют положительные меры Лебега в $\mathbb{R}^{n}$. Таким образом, при малых $\varepsilon$ уравнение задачи (1) вырождается на множестве $\Omega_{0}^{\varepsilon}$, моделирующем блоки.

Такая зависимость от малого параметра и приводит к осредненной задаче в свертках, решения которой приближают решение задачи (1) при малых $\varepsilon$ в соответствии с [1-5]. Для точной формулировки таких осредненных начально-краевых задач понадобятся дополнительные определения.

Пусть вектор-функция $N(y)$ является 1-периодическим решением следующей задачи Неймана на на $Y_{1}$ :

$$
-\operatorname{div}_{y}\left(A_{1} \nabla_{y} N\right)=\operatorname{div}_{y} A_{1} \quad \text { в } \quad Y_{1}, \quad-\left(A_{1} \nabla_{y} N, \Upsilon\right)=\left(A_{1}, \Upsilon\right) \quad \text { на } \quad \Gamma_{1},
$$

где $\Upsilon$ обозначает внешнюю нормаль к границе $\Gamma_{1}$. Далее, введем следующие обозначение для матрицы с постоянными компонентами

$$
A=\left|Y_{1}\right|^{-1} \int_{Y_{1}}\left(A_{1}+A_{1} \nabla_{y} N(y)\right) d y,
$$

где $\left|Y_{1}\right|$ обозначает меру Лебега множества $Y_{1}$. Известно [18], что такая матрица определена и является симметричной и эллиптической. 
Пусть, кроме того, функция $q(t, y)$ является 1-периодическим решением начально-краевой задачи на $Y_{0}$ :

$$
q_{t}^{\prime}-\operatorname{div}_{y}\left(A_{0} \nabla_{y} q\right)=0 \quad \text { в } \quad Y_{0} \times(0, \infty),\left.\quad q\right|_{t=0}=1,\left.\quad q\right|_{\partial Y_{0} \times(0, \infty)}=0 .
$$

Известно [18], что подходящее решение этой задачи существует и функция

$$
m(t)=\left|Y_{1}\right|^{-1} \int_{Y_{0}} q_{t}^{\prime}(t, y) d y
$$

определена как элемент пространства $L^{1}(0, \infty)$ в соответствии с $[2-5]$.

Осредненная задача в свертках для функции $v=v(t, x)$ имеет вид

$$
\begin{gathered}
v_{t}^{\prime}-m *\left(v_{t}^{\prime}\right)-\operatorname{div} A(\nabla v)=f-m * f \quad \text { в } \quad \Omega \times(0, \infty), \\
\left.v\right|_{t=0}=w \quad \text { в } \Omega, \quad v=0 \quad \text { на } \partial \Omega \times(0, \infty),
\end{gathered}
$$

где $*$ обозначает оператор свертки по $t$, например,

$$
m *\left(v_{t}^{\prime}\right)=\int_{0}^{t} m(t-\tau)\left[v_{\tau}^{\prime}(\tau, x)\right] d \tau .
$$

Для фиксированного $\varepsilon$ единственное решение задачи (1) существует, например, в соответствии с [13], [18]. Решение задачи (4) приближает решение задачи (1) в подходящем смысле [1-5] при малых $\varepsilon$. Таким образом, присутствие слабо пористых блоков в области $\Omega$ моделируется появлением «памяти» по плотности (коэффициента при временной производной) у «осредненной» среды. Здесь будет исследована разрешимость задачи (4) при общих исходных данных. Матрица $A$ в уравнении задачи (1) является эллиптической. Поэтому, найдется такое линейное невырожденное преобразование координат в области $\Omega$, что эта матрица перейдет в единичную, что и предполагается в дальнейшем. Кроме того, для упрощения обозначений можно также считать единичной матрицу $A_{0}$ в уравнении задачи (2).

Основным результатом этой работы является следующее утверждение.

Теорема 1. Для каљждого $f \in L^{2}\left(0, \infty ; H^{-1}(\Omega)\right) u w \in L^{2}(\Omega)$ единственное решение $v \in L^{2}\left(0, \infty ; H_{0}^{1}(\Omega)\right)$ задачи (4) существует и найдется такая положительная постоянная $C$, зависящие от $\Omega,\left|Y_{0}\right| u\left|Y_{1}\right|$, что

$$
\begin{gathered}
\|v\|_{L^{2}\left(0, \infty ; H_{0}^{1}(\Omega)\right)}+\left\|v_{t}^{\prime}\right\|_{L^{2}\left(0, \infty ; H^{-1}(\Omega)\right)} \leq \\
\leq C\|f\|_{L^{2}\left(0, \infty ; H^{-1}(\Omega)\right)}+C\|w\|_{L^{2}(\Omega)}
\end{gathered}
$$

$u v \in C^{0}\left([0, T] ; L^{2}(\Omega)\right)$ для фиксированного положительного $T$.

\section{2. ПРЕОБРАЗОВАНИЕ ЛАПЛАСА И ФУНКЦИОНАЛЬНЫЕ ПРОСТРАНСТВА}

Фиксируем $\gamma$ и определим пространство $L_{\gamma}^{2}\left(0, \infty ; L^{2}(\Omega)\right)$ как множество функций из $L_{l o c}^{2}\left(0, \infty ; L^{2}(\Omega)\right)$ для которых конечна величина

$$
\|u\|_{L_{\gamma}^{2}\left(0, \infty ; L^{2}(\Omega)\right)}=\left\|e^{-\gamma t} u\right\|_{L^{2}\left(0, \infty ; L^{2}(\Omega)\right)} .
$$

Последнее равенство задает норму в пространстве $L_{\gamma}^{2}\left(0, \infty ; L^{2}(\Omega)\right)$, относительно которой это пространство является полным в соответствии с [17]. 
Следуя [17], определим пространство $E_{\gamma}\left(L^{2}(\Omega)\right)$ как множество функций $U(\rho)=U\left(\rho_{1}+i \rho_{2}\right)$ со значениями в $L^{2}(\Omega)$, непрерывных и голоморфных в комплексной полуплоскости

$$
\mathbb{C}_{\gamma}=\left\{\rho \in \mathbb{C}: \rho=\rho_{1}+i \rho_{2}, \rho_{1}>\gamma\right\},
$$

для которых конечна величина

$$
\|U\|_{E_{\gamma}\left(L^{2}(\Omega)\right)}^{2}=\int_{-\infty}^{\infty}\left\|U\left(\gamma+i \rho_{2}\right)\right\|_{L^{2}(\Omega)}^{2} d \rho_{2},
$$

где интеграл понимается как предел в среднем интеграла от $K$ до $-K$ при $K \rightarrow \infty$. Последнее равенство определяет норму в пространстве $E_{\gamma}\left(L^{2}(\Omega)\right)$. Следующий вариант теоремы Пэли-Винера доказан в [17].

Теорема 2. Для фиксированного $\gamma$, преобразование Лапласа

$$
\widehat{u(t)}=\int_{0}^{\infty} e^{-\rho t} u(t) d t=U(\rho)
$$

отображает пространство $L_{\gamma}^{2}\left(0, \infty ; L^{2}(\Omega)\right)$ на пространство $E_{\gamma}\left(L^{2}(\Omega)\right)$ взаимно однозначно и взаимно непрерывною.

Аналогично определяются пространства $E_{\gamma}\left(H_{0}^{1}(\Omega)\right)$ и $E_{\gamma}\left(H^{-1}(\Omega)\right)$, для которых также выполнен аналог теоремы 2. Кроме того [17], преобразование Лапласа коммутирует с дифференцированиями по пространственным переменным и оператор свертки по $t$ переводится преобразованием Лапласа в оператор поточечного умножения относительно $\rho \in \mathbb{C}_{\gamma}$.

Будем обозначать $V=\widehat{v}, M=\widehat{m}, Q=\widehat{q}$ и $F=\widehat{f}$. Применяя преобразование Лапласа к (4), получаем

$$
\rho(1-M) V-\Delta V=\mathbb{F} \quad \text { в } \quad \Omega,\left.\quad V\right|_{\partial \Omega}=0,
$$

где

$$
\mathbb{F}=F(1-M)+w(1-M) .
$$

Для фиксированного $\rho \in \mathbb{C}$, задача (5) является краевой задачей для эллиптического уравнения с комплексными коэффициентами в младших слагаемых. Поэтому [17], такая задача разрешима для всех $\rho \in \mathbb{C}$ за исключением, быть может, дискретного множества в $\mathbb{C}$. Здесь, для доказательства разрешимости задачи (4), достаточно будет отделиться от этого множества с помощью априорных оценок с постоянными, не зависящими от $\rho \in \mathbb{C}_{0}$.

\section{3. АПРИОРНЫЕ ОЦЕНКИ РЕШЕНИЙ}

Преобразование Лапласа от задачи (2) имеет вид

$$
\rho Q-\Delta Q=1 \quad \text { в } \quad Y_{0},\left.\quad Q\right|_{\partial Y_{0}}=0 .
$$

Умножая это уравнение на $\bar{\rho} \bar{Q}$ и интегрируя по $Y_{0}$, получаем

$$
\int_{Y_{0}}|\rho Q|^{2} d y+\bar{\rho} \int_{Y_{0}}|\nabla Q|^{2} d y=\int_{Y_{0}} \bar{\rho} \bar{Q} d y .
$$


Аналогично, умножая сопряженное к (7) уравнение на $\rho Q$, имеем

$$
\int_{Y_{0}}|\rho Q|^{2} d y+\rho \int_{Y_{0}}|\nabla Q|^{2} d y=\int_{Y_{0}} \rho Q d y .
$$

Складывая это равенство с (8) и учитывая, что $\rho+\bar{\rho}=2 \rho_{1}$, получаем

$$
\|\rho Q\|_{L^{2}\left(Y_{0}\right)}^{2}+\rho_{1}\|\nabla Q\|_{L^{2}\left(Y_{0}\right)}^{2}=\operatorname{Re} \int_{Y_{0}}(\rho Q) d y .
$$

Поэтому, применяя неравенство Коши-Буняковского к правой части, имеем

$$
\begin{gathered}
\operatorname{Re} \int_{Y_{0}}(\rho Q) d y \leq \int_{Y_{0}}|\rho Q| d y \leq \\
\leq\|\rho Q\|_{L^{2}\left(Y_{0}\right)}\left|Y_{0}\right|^{1 / 2} \leq(1 / 2)\|\rho Q\|_{L^{2}\left(Y_{0}\right)}^{2}+(1 / 2)\left|Y_{0}\right| .
\end{gathered}
$$

Таким образом, заключаем, что

$$
\|\rho Q\|_{L^{2}\left(Y_{0}\right)}^{2} \leq\left|Y_{0}\right|
$$

Из определений и равенства (3) следует, что

$$
M\left|Y_{1}\right|=\int_{Y_{0}}(\rho Q(\rho, y)-1) d y
$$

и

$$
|M|\left|Y_{1}\right| \leq \int_{Y_{0}}|\rho Q-1| d y \leq\|\rho Q\|_{L^{2}\left(Y_{0}\right)}\left|Y_{0}\right|^{1 / 2}+\left|Y_{0}\right| \leq 2\left|Y_{0}\right|
$$

в силу (9). Поэтому, функция $M$ является ограниченной на $\mathbb{C}_{0}$, поскольку непосредственно проверяется, что задача (7) однозначно разрешима для каждого $\rho \in \mathbb{C}_{0}$ и такое решение голоморфно в силу теоремы 2 и известных свойств решений задачи (2), установленных, например, в [13].

Докажем также, что функции $\operatorname{Re}(\rho M)$ и $\operatorname{Re}(M)$ неположительны на $\mathbb{C}_{0}$. Умножая уравнение (7) на $\rho \bar{\rho} \bar{Q}$ и интегрируя по $Y_{0}$, имеем

$$
\rho \int_{Y_{0}} \bar{\rho} \bar{Q}(\rho Q-1) d y+\int_{Y_{0}}|\rho \nabla Q|^{2} d y=0
$$

и

$$
\rho \int_{Y_{0}}(\bar{\rho} \bar{Q}-1)(\rho Q-1) d y+\rho \int_{Y_{0}}(\rho Q-1) d y+\|\rho \nabla Q\|_{L^{2}\left(Y_{0}\right)}^{2}=0 .
$$

Следовательно, используя (10), получаем

$$
-\rho M\left|Y_{1}\right|=\rho\|\rho Q-1\|_{L^{2}\left(Y_{0}\right)}^{2}+\|\rho \nabla Q\|_{L^{2}\left(Y_{0}\right)}^{2} .
$$

Аналогично, учитывая, например, что $\|\rho Q-1\|_{L^{2}\left(Y_{0}\right)}^{2}=\|\bar{\rho}-1\|_{L^{2}\left(Y_{0}\right)}^{2}$ имеем

$$
-\bar{\rho} \bar{M}\left|Y_{1}\right|=\bar{\rho}\|\rho Q-1\|_{L^{2}\left(Y_{0}\right)}^{2}+\|\rho \nabla Q\|_{L^{2}\left(Y_{0}\right)}^{2} .
$$

Таким образом, используя суммирование, из (11) и (12) заключаем, что

$$
\operatorname{Re}(-\rho M) \geq 0, \quad \operatorname{Re}(-M) \geq 0 \quad \text { для } \rho \in \mathbb{C}_{0},
$$

где, для проверки последнего неравенства, следует умножить (11) и (12) на $1 / \rho=\rho_{1} /|\rho|^{2}-i \rho_{2} /|\rho|^{2}$ и $1 / \bar{\rho}=\rho_{1} /|\rho|^{2}+i \rho_{2} /|\rho|^{2}$, соответственно. 
Умножая уравнение $(5)$ на $\bar{V}$ и интегрируя по $\Omega$, получаем

$$
\rho(1-M) \int_{\Omega}|V|^{2} d x+\int_{\Omega}|\nabla V|^{2} d x=\int_{\Omega} \mathbb{F} \bar{V} d x .
$$

Аналогично, умножая сопряженное к (5) уравнение на $V$, имеем

$$
\bar{\rho}(1-\bar{M}) \int_{\Omega}|V|^{2} d x+\int_{\Omega}|\nabla V|^{2} d x=\int_{\Omega} \overline{\mathbb{F}} V d x .
$$

Складывая это равенство с (14) и учитывая (13), получаем

$$
\rho_{1}\|V\|_{L^{2}(\Omega)}^{2}+\|V\|_{H_{0}^{1}(\Omega)}^{2} \leq \operatorname{Re} \int_{\Omega}(\mathbb{F} \bar{V}) d x \leq\|\mathbb{F}\|_{H^{-1}(\Omega)}\|V\|_{H_{0}^{1}(\Omega)} .
$$

Таким образом, заключаем, что

$$
\|V\|_{H_{0}^{1}(\Omega)} \leq\|\mathbb{F}\|_{H^{-1}(\Omega)}
$$

\section{4. РАЗРЕШИМОСТЬ ОСРЕДНЕННЫХ ЗАДАЧ В СВЕРТКАХ}

Учитывая ограниченность $M$, представление (6), неравенства Фридрихса и (15), теорему 2 и следуя [17], получаем следующее утверждение.

Теорема 3. Для каждого $\rho \in \mathbb{C}_{0}, w \in H^{-1}(\Omega)$ u $f \in L^{2}\left(0, \infty ; H^{-1}(\Omega)\right)$ единственное решение $V \in H_{0}^{1}(\Omega)$ задачи (5) существует и найдется такая постоянная $C$, зависящая только от размера $\Omega,\left|Y_{0}\right| u\left|Y_{1}\right|$, что

$$
\|V\|_{H_{0}^{1}(\Omega)} \leq C\|w\|_{H^{-1}(\Omega)}+C\|F\|_{H^{-1}(\Omega)} .
$$

Решение задачи (5) имеет также и некоторые дополнительные свойства.

Лемма 1. Решение задачи (5) непрерывно на $\mathbb{C}_{0}$.

Доказательство. Фиксируем $\rho_{0} \in \mathbb{C}_{0}$ и пусть $\rho \rightarrow \rho_{0}$. Введем обозначение

$$
P(\rho)=\rho(1-M(\rho)) .
$$

В таком случае, задачи $(5)$ в точках $\rho$ и $\rho_{0}$ запишутся в виде

$$
\begin{gathered}
-\Delta V(\rho)+P(\rho) V(\rho)=\mathbb{F}(\rho),\left.\quad V(\rho)\right|_{\partial \Omega}=0, \\
-\Delta V\left(\rho_{0}\right)+P\left(\rho_{0}\right) V\left(\rho_{0}\right)=\mathbb{F}\left(\rho_{0}\right),\left.\quad V\left(\rho_{0}\right)\right|_{\partial \Omega}=0 .
\end{gathered}
$$

Обозначим также $V_{0}(\rho)=V(\rho)-V\left(\rho_{0}\right)$. Вычитая приведенные уравнения и замечая, что $P\left(\rho_{0}\right)=P(\rho)+\left(P\left(\rho_{0}\right)-P(\rho)\right)$, получаем

$$
-\Delta V_{0}(\rho)+P(\rho) V_{0}(\rho)=\mathbb{F}(\rho)-\mathbb{F}\left(\rho_{0}\right)-\left(P(\rho)-P\left(\rho_{0}\right)\right) V\left(\rho_{0}\right) .
$$

Таким образом, повторяя доказательство неравенства (16), имеем

$$
\begin{gathered}
\left\|V_{0}\right\|_{L^{2}(\Omega)}+\left\|V_{0}\right\|_{H_{0}^{1}(\Omega)} \leq C\left\|\mathbb{F}(\rho)-\mathbb{F}\left(\rho_{0}\right)\right\|_{H^{-1}(\Omega)}+ \\
+\left|P(\rho)-P\left(\rho_{0}\right)\right| C\left\|V\left(\rho_{0}\right)\right\|_{H^{-1}(\Omega)} \rightarrow 0
\end{gathered}
$$

при $\rho \rightarrow \rho_{0}$, поскольку $\mathbb{F}(\rho)$ и $P(\rho)$ непрерывны в силу теоремы 2.

Лемма 2. Решение задачи (5) голоморфно на $\mathbb{C}_{0}$. 
Доказательство. Дифференцируя по $\rho$ задачу (5) формально, получаем

$$
-\Delta V_{\rho}^{\prime}(\rho)+P(\rho) V_{\rho}^{\prime}(\rho)=\mathbb{F}_{\rho}^{\prime}(\rho)-P_{\rho}^{\prime}(\rho) V(\rho),\left.\quad V_{\rho}^{\prime}(\rho)\right|_{\partial \Omega}=0 .
$$

Как и при доказательстве теоремы 3 , проверяется, что единственное решение задачи (18) существует и удовлетворяет оценке, аналогичной (16). Здесь следует учесть, что $P_{\rho}^{\prime}(\rho)$ ограничено на $\mathbb{C}_{0}$. Последнее утверждение проверяется дифференцированием по $\rho$ задачи (7) и повторением доказательства неравенства (9) для решений полученной задачи.

Действительно, из определений и представления (10) следует, что

$$
P_{\rho}^{\prime}=1-M+\rho \int_{Y_{0}}\left(\rho Q_{\rho}^{\prime}(\rho, y)+Q(\rho, y)\right) d y,
$$

где первые два слагаемых справа ограничены. Используя (7), имеем

$$
\rho\left(\rho Q_{\rho}^{\prime}+Q\right)-\Delta\left(\rho Q_{\rho}^{\prime}+Q\right)=1-\rho Q \quad \text { в } \quad Y_{0},\left.\quad Q_{\rho}^{\prime}\right|_{\partial Y_{0}}=0 .
$$

Умножая это уравнение на $\bar{\rho}\left(\bar{\rho} \bar{Q}_{\rho}^{\prime}+\bar{Q}\right)$ и интегрируя по $Y_{0}$, получаем

$$
\left\|\rho\left(\rho Q_{\rho}^{\prime}+Q\right)\right\|_{L^{2}\left(Y_{0}\right)}^{2}+\bar{\rho}\left\|\nabla\left(\rho Q_{\rho}^{\prime}+Q\right)\right\|_{L^{2}\left(Y_{0}\right)}^{2}=\int_{Y_{0}}(1-\rho Q) \bar{\rho}\left(\bar{\rho} \bar{Q}_{\rho}^{\prime}+\bar{Q}\right) d y .
$$

Добавляя к этому равенству сопряженное, заключаем, что

$$
\left\|\rho\left(\rho Q_{\rho}^{\prime}+Q\right)\right\|_{L^{2}\left(Y_{0}\right)} \leq\|\rho Q-1\|_{L^{2}\left(Y_{0}\right)} .
$$

Откуда и (9) следует ограниченность $P_{\rho}^{\prime}(\rho)$. Также непосредственно проверяется голоморфность этой функции, поскольку $\rho Q$ голоморфно.

Фиксируем $\rho_{0} \in \mathbb{C}_{0}$ и пусть $\rho \rightarrow \rho_{0}$ для $\rho \neq \rho_{0}$. Умножая уравнение из 17) на $\left(\rho-\rho_{0}\right)^{-1}$ и вычитая из полученного соотношения уравнение из $(18)$, рассматриваемое в точке $\rho_{0}$, имеем

$$
-\Delta V_{r}(\rho)+P(\rho) V_{r}(\rho)=\mathbb{F}_{r}(\rho)-P_{r}(\rho) V(\rho),\left.\quad V_{r}(\rho)\right|_{\partial \Omega}=0,
$$

где, например, обозначено

$$
V_{r}(\rho)=\left(V(\rho)-V\left(\rho_{0}\right)\right)\left(\rho-\rho_{0}\right)^{-1}-V_{\rho}^{\prime}\left(\rho_{0}\right) .
$$

Следовательно, повторяя доказательство неравенства (16), имеем

$$
\begin{gathered}
\left\|V_{r}\right\|_{L^{2}(\Omega)}+\left\|V_{r}\right\|_{H_{0}^{1}(\Omega)} \leq C\left\|\mathbb{F}_{r}(\rho)\right\|_{H^{-1}(\Omega)}+ \\
+\left|P_{r}(\rho)\right| C\left\|V\left(\rho_{0}\right)\right\|_{H^{-1}(\Omega)} \rightarrow 0
\end{gathered}
$$

при $\rho \rightarrow \rho_{0}$, поскольку $\mathbb{F}(\rho)$ и $P(\rho)$ голоморфны в силу теоремы 2 .

Таким образом, учитывая интегрируемость $\mathbb{F}(\rho)$ из (6) в смысле определения пространства $E_{0}\left(H^{-1}(\Omega)\right)$ при $w=0$, получаем такое утверждение.

Теорема 4. Для каждого $f \in L^{2}\left(0, \infty ; H^{-1}(\Omega)\right)$ u $w=0$ единственное решение $v \in L^{2}\left(0, \infty ; H_{0}^{1}(\Omega)\right)$ задачи (4) существует и найдется такая постоянная $C$, зависящая только от размера $\Omega,\left|Y_{0}\right| u\left|Y_{1}\right|$, что

$$
\|v\|_{L^{2}\left(0, \infty ; H_{0}^{1}(\Omega)\right)} \leq C\|f\|_{L^{2}\left(0, \infty ; H^{-1}(\Omega)\right)} .
$$


Используя линейность задачи (4), для завершения исследования разрешимости осредненных задач остается рассмотреть случай произвольного $w$ и $f=0$. В таком случае, условие интегрируемости $\mathbb{F}(\rho)$ из $(6)$ в смысле определения пространства $E_{0}\left(H^{-1}(\Omega)\right)$ не выполняется. Однако, можно воспользоваться также линейностью задачи (4) и известными оценками [13].

Рассмотрим следующую вспомогательную задач для функции $\tilde{v}=\tilde{v}(t, x)$ :

$$
\begin{gathered}
\tilde{v}_{t}^{\prime}-\Delta \tilde{v}=0 \quad \text { в } \quad \Omega \times(0, \infty), \\
\left.\tilde{v}\right|_{t=0}=w \quad \text { в } \Omega, \quad \tilde{v}=0 \quad \text { на } \partial \Omega \times(0, \infty) .
\end{gathered}
$$

Известно, что единственное решение $\tilde{v} \in L^{2}\left(0, \infty ; H_{0}^{1}(\Omega)\right)$ этой задачи существует и $\tilde{v}_{t}^{\prime} \in L^{2}\left(0, \infty ; H^{-1}(\Omega)\right)$. Действительно, умножая на $\tilde{v}$ уравнение задачи $(20)$ в $L^{2}(\Omega)$ и следуя [13], получаем

$$
\left(\int_{\Omega} \tilde{v}^{2} d x\right)_{t}^{\prime}+2 \int_{\Omega}(\nabla \tilde{v})^{2} d x=0 .
$$

Интегрируя это равенство по $t$ заключаем, что

$$
2 \int_{0}^{t}\|\tilde{v}\|_{H_{0}^{1}(\Omega)}^{2} \leq\|w\|_{L^{2}(\Omega)}^{2} .
$$

Следовательно, переходя к пределу по $t$, имеем $\tilde{v} \in L^{2}\left(0, \infty ; H_{0}^{1}(\Omega)\right)$.

Поэтому, для решения задачи (20) выполнено включение

$$
\tilde{v}_{t}^{\prime}=\Delta \tilde{v} \in L^{2}\left(0, \infty ; H^{-1}(\Omega)\right) .
$$

При сделанных предположениях для $w$ и $f$, введем обозначение

$$
u=v-\tilde{v},
$$

где $v$ и $\tilde{v}$ решения задач (4) и (20). Тогда $u$ является решением задачи

$$
\begin{gathered}
u_{t}^{\prime}-m *\left(u_{t}^{\prime}\right)-\Delta u=m *\left(\tilde{v}_{t}^{\prime}\right) \quad \text { в } \quad \Omega \times(0, \infty), \\
\left.u\right|_{t=0}=0 \quad \text { в } \Omega, \quad u=0 \text { на } \partial \Omega \times(0, \infty) .
\end{gathered}
$$

Таким образом, повторяя доказательство теоремы 4 для этой задачи, получаем следующее утверждение.

Теорема 5. Для каждого $w \in L^{2}(\Omega)$ u $f=0$ решение $v \in L^{2}\left(0, \infty ; H_{0}^{1}(\Omega)\right)$ задачи (4) существует, единственно и найдется такая положительная постоянная $C$, зависящая только от размера $\Omega,\left|Y_{0}\right| u\left|Y_{1}\right|$, что

$$
\|v\|_{L^{2}\left(0, \infty ; H_{0}^{1}(\Omega)\right)} \leq C\|w\|_{L^{2}(\Omega)} .
$$

Далее, для $f$ и $w$ из теоремы 1 перепишем задачу (5) в следующем виде

$$
\rho V-w=F+(1-M)^{-1} \Delta V \quad \text { в } \Omega,\left.\quad V\right|_{\partial \Omega}=0,
$$

где функция $(1-M)^{-1}$ определена, поскольку из (9) и (13) следует, что

$$
1 \leq 1-\operatorname{Re} M \leq|1-\operatorname{Re} M| \leq|1-M| \leq 1+|M| \leq 1+2\left|Y_{0}\right|\left|Y_{1}\right|^{-1} .
$$

Для комплексного $\rho=\rho_{1}+i \rho_{2}$ имеем $\rho^{-1}=\rho_{1}|\rho|^{-2}-i \rho_{2}|\rho|^{-2}$ и поэтому

$$
\left|(1-M)^{-1}\right| \leq|1-\operatorname{Re} M|+|\operatorname{Im} M| \leq 1+2|M| \leq 1+4\left|Y_{0}\right|\left|Y_{1}\right|^{-1} .
$$

Следовательно, функция $(1-M)^{-1}$ ограничена на $\mathbb{C}_{0}$. 
Для $f \in L^{2}\left(0, \infty ; H^{-1}(\Omega)\right)$ и $w \in L^{2}(\Omega)$ правая часть уравнения из $(21)$ принадлежит $H^{-1}(\Omega)$ для $\rho \in \mathbb{C}_{0}$ и интегрируема в смысле определения $E_{0}\left(L^{2}(\Omega)\right)$. Кроме того, преобразование Лапласа от производной $v_{t}^{\prime}$ решения задачи (4) совпадает с левой частью этого уравнения.

Таким образом, найдется такая положительная постоянная $C$, зависящая только от размера $\Omega,\left|Y_{0}\right|$ и $\left|Y_{1}\right|$, что

$$
\left\|v_{t}^{\prime}\right\|_{L^{2}\left(0, \infty ; H^{-1}(\Omega)\right)} \leq C\|w\|_{L^{2}(\Omega)}+C\|f\|_{L^{2}\left(0, \infty ; H^{-1}(\Omega)\right)} .
$$

Фиксируем положительное $T$. В таком случае, выполнены включения $v \in L^{2}\left(0, T ; H_{0}^{1}(\Omega)\right)$ и $v_{t}^{\prime} \in L^{2}\left(0, T ; H^{-1}(\Omega)\right)$. Поэтому, из известной теоремы о вложении, приведенной, например, в [13], получаем $v \in C^{0}\left([0, T] ; L^{2}(\Omega)\right)$, что завершает доказательство теоремы 1.

\section{ЗАКЛЮЧЕНИЕ}

В работе исследованы начально-краевые задачи для нестационарных уравнений диффузии и фильтрации в слабо пористых средах. Получены утверждения о разрешимости таких задач и соответствующих осредненных задач в свертках. Эти утверждения доказаны для общих исходных данных и неоднородных начальных условиях и обобщают классические результаты о разрешимости начально-краевых задач для уравнения теплопроводности. В доказательствах используются методы априорных оценок и известный метод Аграновича-Вишика, основанный на преобразовании Лапласа и разработанный для изучения параболических задач общего типа.

Работа выполнена при финансовой поддержке МОН Украины (проект 0219U008403).

\section{ЛитеРАТУРА}

1. Sandrakov G. V. The homogenization of nonstationary equations with contrast coefficients. Dokl. Mathematics. 1997. Vol. 56:1. P. 586-589.

2. Sandrakov G. V. Homogenization of parabolic equations with contrasting coefficients. Izvestiya: Math. 1999. Vol. 63:5. P. 1015-1061.

3. Sandrakov G. V. Multiphase models of nonstationary diffusion in homogenization. Comput. Math. Math. Phys. 2004. Vol. 44:10. P. 1741-1756.

4. Sandrakov G. V. Homogenization of variational inequalities for non-linear diffusion problems in perforated domains. Izvestiya: Math. 2005. Vol. 69:5. P. 1035--1059.

5. Sandrakov G. V. Multiphase homogenized diffusion models for problems with several parameters. Izvestiya: Mathematics. 2007. Vol. 71:6. P. 1193-1252.

6. Sandrakov G. V. Homogenization of some hydrodynamics problems. Сучасні проблеми математич. моделювання, обчислювальних методів та інформаційних технологій. Матеріали Міжн. наук. конф. 2-4 березня 2018, Рівне. С. 156-157.

7. Sandrakov G. V. Modeling of hydrodynamics processes with phase transition. Information Technologies and Computer Modelling. Proceedings of the International Scientific Conf. 14-19 May 2018, Ivano-Frankivsk. P. 303-306.

8. Sandrakov G. V. Modeling of heterogeneous fluid dynamics with phase transition. Information Technologies in Education, Science and Technology. Proceedings of Int. Scientific-Practical Conf. 17-18 May 2018, Cherkasy. P. 142-143. 
9. Sandrakov G. V. Modeling and homogenization of hydrodynamics processes with the vanishing viscosity. Int. School-Workshop on Differential Equations and Applications. 18-20 June, 2019, Vinnytsia. Book of Abstracts. P. 62-63.

10. Sandrakov G. V. Homogenized models with memory effects for composites. Int. School-Workshop on Differential Equations and Applications. 18-20 June, 2019, Vinnytsia. Book of Abstracts. P. 63-64.

11. Сандраков Г. В. Осредненные модели многофазной диффузии в пористых средах. Журнал обчисл. та прикл. матем. 2019. № 3 (132). С. 43-59.

12. Hulianytskyi A. L. Weak solvability of the variable-order subdiffusion equation. Fractional Calculus and Applied Analysis 2020. Vol. 23:3. P. 920-934.

13. Duvaut G., Lions J.-L. Les inequations en mecanique et en physique. Dunod, Paris 1972.

14. Amosov A. A., Zlotnik A. A. On quasi-averaged equations of the one-dimensional motion of a viscous barotropic medium with rapidly oscillating data. Comput. Math. Math. Phys. 1996. Vol. 36:2. P. 203-220.

15. Amosov A. A., Zlotnik A. A. On the quasi-averaging of a system of equations of the one-dimensional motion of a viscous heat-conducting gas with rapidly oscillating data. Comput. Math. Math. Phys. 1998. Vol. 38:7. P. 1152-1167.

16. Amosov A. A., Zlotnik A. A. Justification of two-scale averaging of equations of one-dimensional nonlinear thermoviscoelasticity with nonsmooth data. Comput. Math. Math. Phys. 2001. Vol. 41:11. P. 1630-1650.

17. Agranovich M. S., Vishik M. I. Elliptic problems with a parameter and parabolic problems of general type. Russian Math. Surveys 1964. Vol. 19:3. P. 53-157.

18. Bakhvalov N. S. and Panasenko G. P. Homogenization: averaging processes in periodic media. Kluwer, Dordrecht 1989.

Поступила: 12.12.2020 / Принята: 18.12.2020

\title{
РОЗВ'ЯЗУВАНІСТЬ ОСЕРЕДНЕНИХ ЗАДАЧ У ЗГОРТКАХ ДЛЯ СЛАБО ПОРИСТИХ СЕРЕДОВИЩ
}

\section{Г. В. САНДРАКОВ, А. Л. ГУЛЯНИЦьКИЙ}

Факультет комп'ютерних наук і кібернетики, Київський національний університет імені Tараса Шевченка, Київ, Україна, E-mail: \{gsandrako, andriyhul\}@gmail.com

\begin{abstract}
АнотАция. Розглянуто початкові крайові задачі для нестаціонарних рівнянь дифузії та фільтрації в слабо пористих середовищах. Наведено твердження про розв'язуваність таких задач та відповідних осереднених задач у згортках. Такі твердження, що є узагальненнями класичних результатів про розв'язуваність початково-крайових задач для рівняння теплопровідності, доведено для загальних початкових даних та неоднорідних початкових умов. В доведеннях використовуються методи апріорних оцінок та відомий метод Аграновича-Вішика, розроблений для вивчення параболічних проблем загального типу.

КЛЮчові СловА: початково-крайові задачі, задачі у згортках, осереднення, осереднені задачі, розв'язуваність, апріорні оцінки, перетворення Лапласа.
\end{abstract}

